\title{
FRIEND OR FOE? The Ambivalent Relationship between Mobile Technology and its Users
}

\author{
Sirkka L. Jarvenpaa \\ Mc Combs School of Business \\ The University of Texas at Austin \\ Austin, TX U.S.A. \\ Karl Reiner Lang \\ Zicklin School of Business \\ Baruch College, City University of New York \\ New York, NY U.S.A. \\ Virpi Kristiina Tuunainen \\ Helsinki School of Economics \\ Helsinki, Finland
}

\begin{abstract}
This paper reports on an empirical study that examined the total user experience of mobile technology users. We held a total of 33 focus group sessions comprised of 222 active mobile device users in four highly developed countries (Finland, Japan, Hong Kong, and the United States) with high penetration of mobile technology. We are specifically focusing on manifestations of paradoxes with regard to mobile technology. We identify eight major technology paradoxes that play a central role in the mobile technology usage experience: (1) empowerment-enslavement, (2) independence--dependence, (3) fulfills needs-creates needs, (4) competence-incompetence, (5) planningimprovisation, (6) engaging-disengaging, (7) public-private, and (8) illusiondisillusion. Our findings suggest conceptualizing the phenomenon of mobile technology usage experience from a context-based and process-oriented perspective where paradoxes of technology shape user experience and determine coping strategies.
\end{abstract}




\section{INTRODUCTION}

Consumers and professionals alike struggle with the complexities of the fastchanging possibilities and limitations of the newly emerging mobile communication and computing technologies (Balasubramanian et al. 2002; Jarvenpaa et al. 2003). Mobility, ubiquity, and personalization are salient characteristics that differentiate mobile technologies from other information technologies. They mean $A A A$ capability, that is, the ability to do anything, anywhere, at anytime. Mobile technology promises total computing and communication support for people on the go, the tool of choice for the modern nomad, moving between professional and social environments while seamlessly connected and engaged with business talk, family affairs, and social matters (Lyytinen and Yoo 2002). This technology-enabled augmentation of human powers has undoubtedly made a positive impact on many people's lives. This is the bright side of the technology coin. But this same coin has another, darker side, one that implies negative consequences for the users of mobile technologies. The very same technology that allows users to reach out at will and communicate and transact with others in turn allows these others to uncontrollably reach in from the outside and infiltrate personal space. In other words, mobile technology also means if I can see you, you can see me and that you cannot have one without the other. A personal mobile device establishes a place in itself, a location that provides a fixed contact point where the physical and the virtual space meet, where the individual self and the Net interact. This ambivalence is specific to mobile technologies. The positive and the negative impacts are conceptually inseparable. They create tension and paradoxes.

This paper presents an empirical study that examines the total usage experience of mobile technology users. We were interested in the manifestations of paradoxes with regard to this particular technology, if and in what form and to what extend they occurred, how they occurred, why they occurred, what impact they had on users, and how users responded to ensuing paradoxical situations. Besides providing a descriptive account of the various user experiences, we also aim to present a useful theoretical framework that helps us to anticipate, explain, and evaluate the different user experiences and consequences that result from the adoption and use of mobile technologies.

In order to better understand the contradictory and ambiguous reality of mobile technology use, we conducted a qualitative research study whose findings suggest conceptualizing the phenomenon of mobile technology usage experience from a contextbased and process-oriented perspective. This view is in accordance with Orlikowski (1993), who argues that in order to account for the experiences and implications associated with the usage of IT one has to consider the specific contexts in which IT is being used as well as how the user experience unfolds over time. Similarly, the user-technology interaction process shapes total user experience, but the dynamics of the interaction process depend not only on the user's own responses to the technology but also, to a large degree, on the other users and their uses of the service as well. We identify eight major technology paradoxes that play a central role in the specific case of mobile technology usage: (1) empowerment-enslavement, (2) independence- dependence, (3) fulfills needs-creates needs, (4) competence-incompetence, (5) planningimprovisation, (6) engaging-disengaging, (7) public-private, and (8) illusiondisillusion. Coping with technology paradoxes emerged as the central theme from our 
data. While users almost universally acknowledged that mobile technology had made some improvements to their lives in terms of convenience, flexibility, connectedness, and new freedoms of choice, it became apparent that their overall experience was, to a large extent, determined by conflict situations they had encountered.

\section{RESEARCH METHODOLOGY}

Focus group research is an appropriate qualitative methodology to gain insights to causes and consequences of frequently occurring phenomena that may be experienced differently by different groups of people. Focus group data may also be used for uncovering theoretical concepts and their relationships between concepts in order to develop new theory. While it cannot scientifically test theory, it is a valid method for deriving scientific knowledge and theory building (Fern 2001).

This research followed the commonly accepted guidelines of focus group research (Fern 2001; Krueger 1994). We held a total of 33 focus group sessions comprised of 222 active mobile device users in four countries (Finland, Japan, Hong Kong, and the United States) with a high penetration of mobile technology devices. While all 33 focus groups were urban-based, the groups varied in age, gender, culture, and economic standing (see Table 1). Data were collected over a 9-month period in 2001. The focus group discussion questions, shown in Table 2, were kept as broad as possible to ensure open forums. Each focus group lasted about 90 minutes to 2 hours and was conducted by a moderator in the local language. The session protocol transcripts were translated and then content analyzed using the principles of the grounded theory approach (Auerbach and Silverstein 2003; Strauss and Corbin 1998).

For the purpose of this paper, we define mobile technology as an information technology artifact that is represented as a service bundle combining a device with its interface, network services, and software applications. Because these are so intertwined it does not make sense to disentangle device, interface, and applications when studying how mobile services create value for the users. This definition includes mobile phones, portable digital assistants, and integrated wireless enterprise solutions like the popular handheld BlackBerry ${ }^{\mathrm{TM}}$.

\section{RESEARCH RESULTS}

Among our focus group participants, voice communication clearly dominates the usage profile, followed by data services like e-mail, Web browsing, and text messaging. Other informational and transactional services were only moderately used on a regular basis. The process of experiencing mobile technology from a user's perspective is depicted in Figure 1, which shows the main themes and concepts that emerged from our data and the relationships that indicate significant interactions between them. This process model is proposed as an initial theoretical framework for anticipating, explaining, and evaluating the experiences and behavioral responses associated with the use of mobile technology. 
Table 1. Focus Group Participants

\begin{tabular}{|l|l|}
\hline \multicolumn{1}{|c|}{ Location } & \multicolumn{1}{|c|}{ Focus Group Participants (Group Size) } \\
\hline \multirow{5}{*}{ Helsinki, Finland } & Airline Maintenance staff (8) \\
& Adult hobby group (7) \\
& Late Teens (6) \\
& Boys, 11-12 years old (12) \\
& Girls, 11-12 years old (8) \\
& Mothers (8) \\
& Software Consultants (6) \\
& Entrepreneurs (8) \\
& Students (6) \\
& Researchers, all female (7) \\
\hline Hong Kong & Undergraduate Students I (7) \\
& University Maintenance Workers, all male (5) \\
& Kitchen/Restaurant Staff (6) \\
& Undergraduate Students II (8) \\
& Rock Band (4) \\
& IT Professionals (4) \\
\hline \multirow{5}{*}{ Tokyo, Japan } & High School Students, all female (6) \\
& Vocational School Students, all female (6) \\
& Housewives (6) \\
& IT Sales Representatives (7) \\
& MBA Students (9) \\
\hline \multirow{5}{*}{ Austin, USA } & Real Estate Agents (7) \\
& Church Group (8) \\
& Engineers, all male (6) \\
& Lawyers, (7) \\
& Graduate Students (7) \\
& IT Professionals (6) \\
& Women's Group (5) \\
& Professionals (7) \\
\hline &
\end{tabular}

Table 2. Focus Group Discussion Questions

1. How do you feel when you have to turn your device off?

2. Why are you using mobile services?

3. What kinds of services are missing? Why do you need them?

4. Why do you use a mobile device rather than a wired device to access a particular service?

5. How do other technologies support your use of mobile services?

6. What is the value of mobile services to you? What is the most valuable thing?

7. What problems and risks are associated with the use of mobile devices?

8. How have/are mobile phones changing your life and affecting the quality of your life? 
The model in Figure 1 does not explain why or how consumers decided to acquire mobile technology. That is, the adoption decision is treated as an exogenous prior event. The actions and experiences of mobile technology users depend on situational and contextual factors. The given technology, social (personal or organizational), and cultural contexts influence user's motivations, goals, and usage patterns when using mobile technology for a particular purpose (arrow 1). Decisions to use mobile technology in different situations for different purposes provide accumulative contextfeedback over time, which can change or reinforce those contexts (arrow 2). But no matter what the specific purpose might be, eventually and recurrently, technology use will create conflict situations for the user, although the specific nature of the conflict may differ between, for example, maintaining social relationships and performing business tasks (arrow 3). Again, context-dependent (arrow 4) conflict situations will arise from paradoxes that are inherently and systemically linked to technology use.

The repeated confrontation with paradoxically behaving technology impacts users on an emotional level. This has a significant effect on the total user experience. The continuous conflict with paradoxes and emotional responses leads, for many long-term users, to a search for identity as they keep participating in multiple realities, moving simultaneously in loosely connected virtual spaces and the physical space, a search that is typically accompanied by attempts of reconstructing the meaning of traditional social values and concepts. Reinforcing or modifying context-feedback is generated as users experience conflict (arrow 5).

A direct response to the challenges presented is the development of behavioral strategies that help users better cope with these conflict situations (arrow 6). Broadly speaking, users work out and adopt mechanisms that help them avoid or minimize conflict (avoidance strategies) or guide them in confronting and perhaps managing conflict (confrontative strategies). The particular reactions of users and their ability to manage conflict and to cope with the technology challenge are once more influenced by situational context factors (arrow 7). Context-feedback is generated as users gain experience with coping strategies (arrow 8).

\section{MOBILE TECHNOLOGY PARADOXES AND CONFLICT SITUATIONS IN THE USER'S EXPERIENCE}

This section describes in more detail, based on the data collected from our focus group sessions, the various manifestations of technology paradoxes (depicted in Figure 1) in the specific context of mobile technology. It discusses why paradoxes occur, how they occur, and how users respond to them. Eight central paradoxes emerged from our data. They are presented in order, starting out with paradoxes that occur primarily due to individual behavior and following with those that are increasingly determined by group behavior. The described paradoxes don't happen in isolation, they may overlap and interact with each other. 


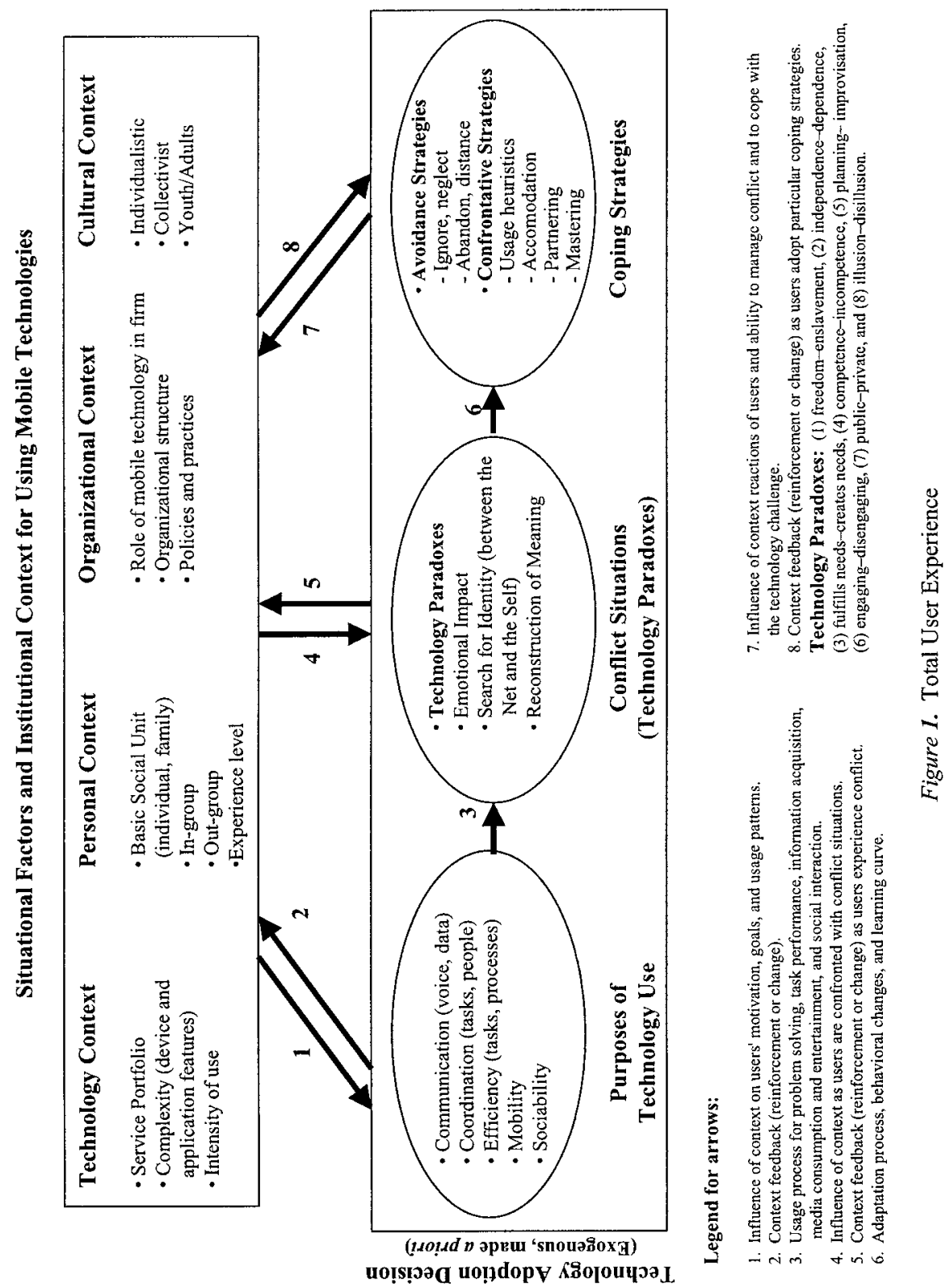




\subsection{The Empowerment-Enslavement Paradox}

New freedoms of choice surfaced as the perhaps most salient issue from mobile technology use across all types of users. Nearly everyone praised some new possibilities that were related to the ubiquitous, 24/7, "always on" nature of mobile technology. Permanent connectivity allows people to take charge anytime wherever they are, whether it concerns business, family, or friends. This newly found freedom empowers users, but the very same connectivity also prevents users from creating and maintaining distance from others. "The fact that you have to interact with these devices is bad," explained one user. Aside from concerns about increased surveillance by government and corporate institutions using GPS, it has generally become difficult to get away from people you would rather not communicate with. An older woman reminisced old times, "when we did not have any of these technologies. Doing any kind of job was really hard back then. I can tell you that the cell phone really is a tremendous safety and access device. But I should be controlling it, not it controlling me." The unpredictability and uncertainty if and when a call may come and demand unwanted attention counteracts the power that users derive from the technology. "I am in a dilemma that I cannot leave my cell phone at home, but just the fact that I am always connected is stressful," remarked one person. And someone else added, "Availability all the time! This is not what we humans were made for." Some users had a pretty good sense of the power they could exert but also anticipated that it could backfire in just the same way. One participant observed, "The mobile is great for controlling other people like your wife and kids, for example. That would be pretty good if I had the choice to decide who knows where I am and where I have been." Many users reported great pressures and felt forced to respond to the technology, whether they wanted to or not. Some feared that they had become slaves to the machine.

The workplace was another area where the empowerment-enslavement paradox manifested itself on a regular basis. Most professionals welcomed the introduction of mobile technologies in their companies. They appreciated increased productivity, more flexibility, and more efficient ways to coordinate tasks and people. But again, the same tools that empowered them on their job in so many ways also took away long-cherished freedoms. Increased work pressure, closer monitoring and supervision, and the inability to separate and keep distance from work were cited frequently. Participants expressed displeasure having to play multiple roles at all times, especially having to constantly switch between family and work roles.

\subsection{The Independence-Dependence Paradox}

"My cell phone does everything," said one of our focus group members. More independence through mobility was one of the most important freedoms enjoyed by the participants of our study. But it also became clear that the power to connect independent of space and time created a new form of dependency that invariably coexists with independency. Or as one of our participants described, "Always being available results in lack of independence. It is like having an electronic ankle chain." While the resultant independence-dependence paradox could be considered just a special case of the 
above described empowerment-enslavement paradox, it warrants its own category because this particular aspect was almost universally acknowledged as an issue with which users were struggling. Some denied technology dependency or at least qualified it, but most found it quite difficult to break the always-on habit they had acquired and admitted quite bluntly that they had become dependent on total connectivity. Although "there was life before these gadgets," for most users mobile technology has become part of their lives, for better or worse. Many agreed that "life without a cell would be terrible" and that "once you get one you don't know how to live without it."

Being cut off from her usual mobile services, one participant experienced withdrawal-like symptoms. "Once I went on a trip to [nearby] Macau without my cell phone. Whenever I heard a ring tone I thought someone is calling me. I got so tense and thought it was my phone." Once people get used to being always on it causes them great discomfort when they need to disconnect, even if it is only temporarily. Most users loathe turning off their devices for fear they may miss an important call. Missed calls attain a more meaningful status than most answered calls. The mere chance that someone may have tried to communicate something significant is given substantial consideration despite the fact that most turn out to be noncritical or outright trivial. This leaves users in a state of constant anticipation for some elusive messages that may or may not ever be send. The possibility of failing to immediately respond to either a great opportunity or some emergency is perceived as a great threat, although it is understood, from a rational perspective, that the odds are rather small that an event with significant consequences would be passed over because of a missed call.

\subsection{The Fulfills Needs-Creates Needs Paradox}

Mobile technology has "taken simplicity out of our lives," commented one of our participants. Our data shows clearly that technology really is providing a solution to many problems that benefit from mobile connectivity, but at the same time it has also caused a whole range of new problems, problems that users didn't have before or didn't perceive having. This can be as trivial as the new need of always "having to carry a bag that holds the mobile" or that "the battery runs down quickly," but often it creates much more intricate needs. The irony that solutions create problems did not go unnoticed. As one person urgently expressed, "it is very important that users think about what specific need a particular service is really satisfying," while someone else wondered "if adding more services is really productive? Every time they add something, people have problems." Paradoxically, the same feature that fulfills one user need creates another. For example, mobility fulfills the need for more security because it allows people to stay in touch (e.g., parents with their children when they are out, wives with their husbands when they go on road trips, or elderly with caretakers if they encounter difficulties at home and can't reach the regular phone), but ironically this newly found security also seems to create a new sense of vulnerability as a number of people fear "What if I lose it?"

A new need for privacy was recognized by a number of participants. This need only arose because mobility and $A A A$ capability had fulfilled user's need to be able to talk in public places. But once users experienced situations where they had a conversation in public that they really didn't mean sharing with anyone, and certainly not with random bystanders, or were involuntarily made to listen to some stranger talking on a 
mobile phone, they realized that going public with mobile communication creates a new need for privacy. Most prevalent, however, were cases where users had taken an active step to fulfill a perceived need just to find out that this same action created a new need.

\subsection{The Competence-Incompetence Paradox}

The ability to do anything, anytime, anywhere gives mobile technology users a whole set of new competencies. It allows them to do things they couldn't do before. It enables them to do things more efficiently and effectively than in the past. But as people are using their newly acquired, technology-enabled competencies to perform new tasks or try to perform tasks better, they soon experience a new sense of incompetence. Seemingly simple services turn out to be hard to use and newly gained efficiencies tend to be limited in scope and actually cause inefficiency at some higher level. The idea that a newly obtained competence lowers another, directly related competence or makes users experience incompetence, whether real or just perceived, emerged from our data in several different contexts. The most apparent relates to situations where users explore new device functions or try out new application services with the expectation of becoming a more competent user only to be confronted with unexpected difficulties that leave them feeling less competent than before. Such "less for more" bargains were reported frequently across groups.

Other examples illustrated how the use of one new competence can compromise another. The new competence of talking on the phone while driving can make you a bad and possibly dangerous driver. The new competence of using mobile text communication methods can have a negative effect on your written language competence. Relying on electronic memory may be detrimental to your own memory. Using mobile technologies in meetings may give you better information but may be distractive and actually weaken your performance and thus make you a less competent participant in the meeting.

In many ways, some particular mobile services themselves turn out to be much less competent than expected when applied in varying situations and often outright incompetent when used in ways that are only slightly different from the prescribed guidelines. In either case, mobile technology competencies are too often too narrow in scope and incapable of adapting to specific user environments to be of much value. This leaves the user with the impression that the technology itself is incompetent, or worse, it makes the users themselves feel incompetent as they struggle to make effective use of it.

Poor design or technical limitations like small screen size, tiny input keys, or some network constraints can render a service ineffective and make it look incompetent. On the other hand, complicated usage logic or incomprehensible behavior of a service diminishes self-efficacy and tends to create a sense of user incompetence.

\subsection{The Planning-Improvisation Paradox}

Mobile technologies can certainly be employed as effective planning tools allowing people to better coordinate meetings and plan work and social activities. One user, for example, said, "I use the mobile to be polite and call to tell when I am five minutes late or I may check if the other person is really there where I am supposed to pick him up." 
Taking advantage of $A A A$ capabilities, users can prepare schedules in advance and then update and refine them independent of time and location and provide involved parties with additional information as they go along. This should, in theory, result in more efficient planning and less unproductive time. In practice, however, the opposite takes place all too often. People tend to spend less time and effort on working out schedules and instead rely more on the technology that allows them to make up for lack of preparation with continuous improvisation. Some users welcomed this new flexibility that makes "life more unplanned because you can plan on the spot" and found it liberating that "you don't have to make arrangements in advance anymore." Several participants described situations where vague planning of a meeting lead to numerous changes and improvisations that resulted in extra coordination efforts, modified agendas, and, in the end, increased lateness and actually less time spent together. To some extend, technology substitutes for planning rather than augmenting it.

Technology has been changing people's behavior. It used to be socially unacceptable to be late, without a reasonable excuse, but now being late is almost expected; it has become the norm. As long as you update your party on your whereabouts and report on your progress toward the meeting, being late is acceptable. What is unacceptable, however, is not having a mobile phone and being out of reach. Forgetting the mobile at home or running out of battery have become inexcusable faux pas that can easily result in social exclusion. Another consequence of this emerging improvisation culture is the erosion of social conventions regarding acceptable calling times. In the absence of any preplanning, "It has [for example] become easier to get a hold of a married friend. Since he answers his cell phone, I can call him in the middle of the night without giving any consideration to his family members."

Too much improvisation causes disorganization. While mobile technologies are designed as instruments to support control, they create chaos if improperly used. Technology can facilitate not just planning but also controlling information access and input, but if people don't exercise discipline in their usage, chaos is bound to ensue. Too much communication creates chaotic disturbances in people's physical space; receiving too much information leads to information overload; messages are send and ignored, which triggers more messages and corrupts social communication protocols. Senders no longer know which of their messages have been received and recipients lose messages in an uncontrolled fashion.

\subsection{The Engaging-Disengaging Paradox}

One promise that comes with $A A A$ capability is that mobile technology would enable users to choose when to engage in a discourse and when to disengage, promising tools that help users achieve a balance between involvement and retreat. This desire to retreat from stressful environments while at the same time staying involved surfaced in several of our focus group discussions. For example, one participant expressed that "people want to know what is going on, but on the other hand, they also want to be in the middle of a forest." Unfortunately, most people find it difficult to simultaneously engage in parallel activities, to engage in something new without disengaging from something else. When calls interrupt a conversation in the physical space, the person receiving the call will typically abruptly disengage from the current conversation and 
engage in a new one, often leaving others stranded. When driving, people make calls and engage in conversation while disengaging from their driving activity.

The realization that mobile technologies "might be a detriment to other kinds of communication" was widely acknowledged. Communication patterns in the family home have been altered since the fixed-line family phone has lost its role as the communication hub in the home. "The phone at home does not ring that much anymore since everybody has their own cell phone. When it does ring, it usually is somebody trying to sell us something. No one else calls our home phone anymore." While family members develop their own personal mobile communication channels, they are prone to disengage from family life, especially teenage children. "I would be even less home, if I didn't need to go home to read my e-mail [on the desktop computer]," conceded a young daughter. And parents generally thought that heavy use of mobile technology among teenage kids was detrimental to family life and social interaction.

Generally, there was concern that engaging with mobile communication technologies may lead users to disengage from face-to-face social activities.

\subsection{The Public-Private Paradox}

Mobile technology devices are usually considered personal tools for private communication. In the past, personal communication (voice or data) only took place in personal physical spaces like the office or the home where it was by and large possible to set and control an adequate level of privacy. Now, freed from spatial and temporal constraints, people are increasingly taking private conversations into the public space, which creates friction and interferes with other people's activities and privacy. When exchanging messages or talking, the conversing parties create virtual communication spaces. But while technology can support users in managing multiple virtual communication spaces, it cannot eliminate interference with activities in the surrounding physical space. It is not just the noise and chatter that disturbs people, it is also the fact that they overhear only one half of a nearby conversation, wondering about the missing pieces and the absent person, involuntarily drawn into an interpersonal exchange that should be private but is happening in public. At the same time, people assume different roles as they switch between physical and virtual space, displaying behaviors, gestures, and emotional states that may befit an ongoing virtual interaction but may appear out of place in the context of the present physical space.

\subsection{The Illusion-Disillusion Paradox}

When our users acquired their first mobile devices or upgraded to new models they did so with certain expectations of partaking in joys that would come with $A A A$ capability features that were promised and promoted. Whether these were reasonable or unrealistic, people were anticipating that their new gadgets would make their lives easier and enable them to do things they couldn't before. But soon they learned that true $A A A$ capability represents an ideal that remains an illusion in people's reality and that available technology in many regards delivers merely a crude approximation to their 
initial expectations. Many users recounted frustration and disillusion as they discovered that anywhere communication really means in some places and areas only, while limited coverage and dead spots are really constraining connectivity. "When I tried to send data from the Shinkansen [Japanese bullet train], the transmission was interrupted every time the train went thorough the tunnel," lamented a respondent. Likewise, anytime communication is severely compromised by short battery runtimes. And of course, anytime also requires that the intended communication partners are available and willing to communicate. Finally, anything barely covers voice communication as many connections are unstable or of poor quality while hardware and bandwidth limitations render access to Web sites and delivery of multimedia content inadequate. For example, one user thought "it is a great idea to make comics available over cell phones, but the screen is too tiny. It takes the fun away."

When adopting new mobile services, users were under the impression that they would get to enjoy an upgrade in service level or quality, but after using the new services they often felt that the services were inadequate for the task. They actually perceived a service downgrade. For example, the ability to access the Web with mobile phones promises a service upgrade, but the actual experience of struggling with small keypads and screens and slow connections creates the perception of a downgrade, especially when contrasted with the easily available wired Internet or wireless WiFi networks. "I feel this Internet and mobile thing is like skis that are designed for classical style Nordic skiing and free style Nordic skiing. The middle way may not be the best-some services belong to the Internet and others will be mobile," stated one user. Difficult to use interfaces and cryptic command syntaxes were also significant factors contributing to disappointment about new services. A few people pointed out that more communication does not necessarily mean better communication, and that the ease of communicating with mobile technologies may have increased the quantity of communication but, at the same time, decreased its quality.

\section{DISCUSSION AND CONCLUSION}

The idea that technology is and behaves intrinsically paradoxically or ironically is, of course, not entirely new. For example, Arnold (2003), Castells (2000), and Easterbrook (2003) all argue, from a purely theoretical point of view, that technologies that bring us progress also create economic and social paradoxes that increasingly challenge people in their individual and social lives. Tradeoffs between privacy and awareness and between awareness and disturbance have been recognized as fundamental in the technology design space and are unlikely to be eliminated (Hudson and Smith 1996). In a more business-oriented discourse, Handy (1995) makes the case that the ability to manage paradoxes is key to corporate success in today's high-tech world. Mick and Fournier (1998) describe a post-modernist consumer society in which consumers are confronted with multiple and conflicting consequences from the consumption of consumer electronic products.

Among the information systems researchers, Orlikowski (1991) and Chinn (2001) discuss the paradoxical nature of information technology in general, while Howcroft and Wilson (2003) and Schultze and Orlikowski (2004) specifically examine paradoxes of 
participatory information systems development, which they see as a consequence of inherently conflicting organizational relations. Depending on situational context factors and the particular purposes, mobile technology users are invariably confronted with a set of technology paradoxes that affect their relationship with the technology ambiguously in terms of both emotional impact and behavioral response. In a recursive process, users interact with the properties of mobile technology and come up with their own set of rules that shape how they make use of the technology (Orlikowski 2000).

This research contributes to the understanding of technology paradoxes, and in particular why and how they occur in mobile environments. As indicated in Figure 1, we identify a number of coping mechanisms that can be broadly classified into two groups: avoidance and confrontational coping strategies (Holahan and Moos 1987). The former refers to user strategies that try to minimize interaction with the technology and the latter describes strategies that are based on negotiating with technology. We conclude that managing paradoxes in personal technology spaces arises as a major obstacle in people's lives. This presents both a challenge and an opportunity for technology service providers. New innovations like better presence management or service integration are being developed to address some of the problems discussed in this paper, but at the same time, novel features and new gadgets constantly introduce new complexity and ultimately lead to recurring conflicts. Hence, the elimination of technology paradoxes may proof elusive. On the other hand, we suggest that exploring ways to help users better cope with paradoxically behaving technology is a research direction that needs more attention.

\section{ACKNOWLEDGMENTS}

This project was funded by a grant awarded to the first author by the Advanced Practices Council of the Society for Information Management.

\section{REFERENCES}

Arnold, M. "On the Phenomenology of Technology: The 'Janus-Faces' of Mobile Phones," Information and Organization (13), 2003, pp. 231-256.

Auerbach, C. F., and Silverstein, L. B. Qualitative Data: An Introduction to Coding and Analysis, New York: New York University Press, 2003.

Balasubramanian, S., Peterson, R. A., and Jarvenpaa, S. L. "Exploring the Implications of MCommerce for Markets and Marketing," Journal of the Academy of Marketing Science (30:4), 2002, pp. 348-361.

Castells, M. The Rise of the Network Society. The Information Age: Economy, Society and Culture, Volume 1 ( $2^{\text {nd }}$ ed.), Oxford, UK: Blackwell Publishers, 2000.

Chinn, S. S. "The Technology Paradox," Industrial Management, March-April 2001, pp. 25-27.

Easterbrook, G. The Progress Paradox: How Life Gets Better While People Feel Worse, New York: Random House, 2003.

Fern, E. F. Advanced Focus Group Research, Thousand Oaks, CA: Sage Publications, 2001. Handy, C. The Age of Paradox, Boston: Harvard Business School Press, 1995.

Holahan, C. J., and Moos, R. H. "Personal and Contextual Determinants of Coping Strategies," Journal of Personality and Social Psychology (52:5), 1987, pp. 946-955. 
Howcroft, D., and Wilson, M. "Paradoxes of Participatory Practices: The Janus Role of the Systems Developer," Information and Organization (13), 2003, pp. 1-24.

Hudson, S. E., and Smith, I. "Techniques for Addressing Fundamental Privacy and Disruption Tradeoffs in Awareness Support Systems," in Proceedings of ACM Conference on Computer Supported Collaborative Work, New York: ACM Press, 1996, pp. 248-257.

Jarvenpaa, S. L., Lang, K. R., Takeda, Y., and Tuunainen, V. K. "Mobile Commerce at Crossroads," Communications of the ACM (46:12), 2003, pp. 41-44.

Krueger, R. A. Focus Groups: A Practical Guide for Applied Research, Thousand Oaks, CA: Sage Publications, 1994.

Lyytinen, K., and Yoo, Y. "The Next Wave of Nomadic Computing," Information Systems Research (13:4), 2002, pp. 377-388.

Mick, D. G., and Fournier, S. "Paradoxes of Technology: Consumer Cognizance, Emotions, and Coping Strategies," Journal of Consumer Research (25), 1998, pp. 123-143.

Orlikowski, W. J. "CASE Tools as Organizational Change: Investigating Incremental and Radical Changes in Systems Development," MIS Quarterly (17:3), September 1993, pp. 309-339.

Orlikowski, W. J. "Integrated Information Environment or Matrix of Control? The Contradictory Implications of Information Technology," Accounting, Management, and Information Technology (1), 1991, pp. 1, 9-42.

Orlikowski, W. J. "Using Technology and Constituting Structures: A Practice Lens for Studying Technology in Organizations," Organization Science (11:4), 2000, pp. 404-428.

Schultze, U., and Orlikowski, W. J. "A Practice Perspective on Technology-Mediated Network Relations: The Use of Internet-Based Self-Serve Technologies," Information Systems Research (15:1), 2004, pp. 87-106.

Strauss, A., and Corbin, J. Basics of Qualitative Research, Techniques and Procedures for Developing Grounded Theory, Thousand Oaks, CA: Sage Publications, 1998.

\section{ABOUT THE AUTHORS}

Sirkka L. Jarvenpaa is the Bayless/Rauscher Pierce Refsner Chair in Business Administration and a cofounder and codirector of the Center for Business, Technology, and Law at the McCombs Business School, University of Texas at Austin. Sirkka can be reached at Sirkka.Jarvenpaa@mccombs.utexas.edu.

Karl R. Lang is an associate professor in Information System at the Zicklin School of Business, City University of New York. Karl can be reached at karl_lang@baruch.cuny.edu.

Virpi Kristiina Tuunainen is a professor of Information Systems Science at the department of business technology, and the director of the Graduate School for Electronic Business and Software Industry,Helsinki School of Economics. Virpi can be reached at virpi.tuunainen@, hkkk.fi. 